\title{
No association of TP53 codon 72 and intron 3 16-bp duplication polymorphisms with breast cancer risk in Chinese Han women: new evidence from a population-based case- control investigation
}

Weiming Hao ${ }^{1,2+}, \mathrm{Xia} \mathrm{Xu}^{3 \dagger}$, Haifeng Shi $^{1}$, Chiyu Zhang ${ }^{2}$ and Xiaoxiang Chen ${ }^{4 *}$

\begin{abstract}
Background: Many studies have demonstrated that the genetic variants of tumor suppressor gene TP53 contribute to the prediction of breast cancer risk. However, most of them focused on Europeans and Americans; the investigations about Asians, especially Chinese women, are scarce. Thus, the aim of this study was to explore the influence of TP53 codon 72 and intron 3 16-bp duplication polymorphisms on the breast cancer risk in Chinese women, especially those from eastern China.

Methods: Blood samples collected from 254 breast cancer patients and 252 healthy female individuals were investigated. Genotypes of the two polymorphisms were determined by direct sequencing and conventional PCR, respectively.

Results: Heterozygous Arg/Pro and homozygous Del/Del were the most frequent genotypes of the two polymorphisms, respectively. Heterozygous Arg/Pro had a higher prevalence in breast cancer cases $\left(P_{\text {adj }}=0.10 ; O R_{a d j}=1.43\right.$, 95\% Cl 0.93-2.18), and no homozygous 16-bp duplication (Ins/Ins) genotype was found in the whole 506 clinical samples. For the distributions of allele and haplotype frequencies, no statistically significant difference was observed between the two groups when multiple (additive, dominant and recessive) genetic models were utilized in the analysis $\left(P_{\text {adj }}>0.05\right)$.

Conclusion: The results suggested that the two TP53 polymorphisms did not affect breast cancer risk in Chinese Han women, but the heterozygous Arg/Pro may exist as the possible risk genotype of the codon 72 polymorphism in contrast to the homozygous Arg/Arg and Pro/Pro.
\end{abstract}

Keywords: TP53, Polymorphisms, Breast cancer, Chinese women, Case-control investigation

*Correspondence: cxxxxcyd@gmail.com

tWeiming Hao and Xia Xu contributed equally to this work

${ }^{4}$ Department of Gynecologic Oncology, Jiangsu Cancer Hospital, Jiangsu

Institute of Cancer Research, Nanjing Medical University Affiliated Cancer

Hospital, 42\# Baiziting Street, Nanjing 210009, Jiangsu, People's Republic

of China

Full list of author information is available at the end of the article

(c) The Author(s) 2018. This article is distributed under the terms of the Creative Commons Attribution 4.0 International License (http://creativecommons.org/licenses/by/4.0/), which permits unrestricted use, distribution, and reproduction in any medium, provided you give appropriate credit to the original author(s) and the source, provide a link to the Creative Commons license, and indicate if changes were made. The Creative Commons Public Domain Dedication waiver (http://creativecommons.org/ publicdomain/zero/1.0/) applies to the data made available in this article, unless otherwise stated. 


\section{Background}

Breast cancer is the world's most common and deadly cancer in women. It has been demonstrated that the occurrence of this global disease is associated with not only environmental conditions but also genetic susceptibility $[1,2]$. The tumor suppressor gene TP53, which is located on $17 \mathrm{p} 13.1$ and encodes $p 53$, is one of the most significant breast cancer susceptibility genes, as the $p 53$ protein plays an important role in responding to DNA damage, metabolic stress and oncogene activation [3, 4]. However, many genetic variants, especially single nucleotide polymorphisms (SNPs) in the TP53 gene, have been identified and reported to be cancer associated, since they can result in the changes of amino acids in the DNA-binding domain, and consequently impact the normal functions of $p 53$ protein [5]. Of them, the most extensively studied was codon 72 polymorphism (Arg72Pro, rs1042522), which is caused by the substitution of arginine (Arg) to praline (Pro). A lot of studies supported that Arg72Pro had correlation with risk of different kinds of carcinoma, especially breast cancer [6-15], whereas other studies did not support it [16-23]. Another wellstudied intronic polymorphism is the 16-bp duplication (rs17878362) in intron 3. It was reported that the 16-bp duplication could lead to lower level of p53 transcript, which may provide a possible molecular basis for the association with high risk of cancer [24]. Although many studies have explored its effect on susceptibility of cancers and found some positive results [8, 11, 20, 22-26], the conclusion is conflicting as well. There are also some new advances concerning genetic factors including breast cancer risk in Chinese [27-33] or Asian [34-37] population. More importantly, most of the previous studies on this issue focused on Caucasians living in Europe and America; the statistics about Asians, especially Chinese population, are relatively scarce. Therefore, we carried out a population-based case-control study to investigate the possible association between the two TP53 polymorphisms and breast cancer risk in Chinese Han women.

\section{Methods}

\section{Study population}

A total of 506 unrelated clinical peripheral blood samples including 254 women patients with breast cancer from Jiangsu Cancer Hospital and the other 252 healthy female individuals recruited by Zhenjiang Center for Disease Prevention and Control were collected and detected. All the studied breast cancer cases are sporadic cases, as those belonging to familial cases were excluded based on the Breast Cancer Linkage Consortium criteria (Stratton 1997). The clinicopathological information of the patients and healthy controls is shown in Table 1 . This
Table 1 Clinicopathological information of breast cancer cases and controls

\begin{tabular}{llc}
\hline Group & Clinical data & Number (\%) \\
\hline Controls (N=252, mean age $=41.2 \pm 10.9)$ & \\
Age (years) & $<50$ & $205(81.3)$ \\
& $50-70$ & $45(17.9)$ \\
& $>70$ & $2(0.8)$ \\
Breast cancer cases (N=254, & mean age $=49.5 \pm 9.9)$ & \\
Age (years) & $<50$ & $125(49.2)$ \\
& $50-70$ & $125(49.2)$ \\
Menopausal status & $>70$ & $4(1.6)$ \\
& Premenopausal & $127(50.0)$ \\
& Postmenopausal & $114(44.9)$ \\
Tumor type & NA & $13(5.1)$ \\
& IDC & $221(87.0)$ \\
& ILC & $9(3.5)$ \\
& Others & $12(4.7)$ \\
Tumor stage & NA & $12(4.7)$ \\
& I & $7(2.9)$ \\
& II & $164(67.8)$ \\
& III & $40(16.5)$ \\
& IV & $3(1.2)$ \\
& NA & $28(11.6)$ \\
\hline
\end{tabular}

IDC invasive ductal carcinoma, ILC invasive lobular carcinoma, NA not available

a According to the AJCC Cancer Staging Manual (7th Edition)

study was approved by the Ethics Committee of Jiangsu Cancer Hospital. Informed consent was obtained from all involved participants and publication.

\section{Genomic DNA extraction and TP53 polymorphisms detection}

Genomic DNA (gDNA) was extracted from the blood samples using QIAamp DNA Blood Mini Kit (QIAGEN, Hilden, Germany). The codon 72 polymorphism was determined through direct sequencing. Genomic DNA of each sample was amplified using the forward primer: 5'-GACCTGGTCCTCTGACTGCTCT-3' and reverse primer: $\quad 5$ '-TGACAGGAAGCCAAAGGGTGAAGA G-3'. The PCR thermal cycling conditions were pre-denaturation at $94{ }^{\circ} \mathrm{C}$ for $2 \mathrm{~min}$; 30 cycles of denaturation at $98{ }^{\circ} \mathrm{C}$ for $10 \mathrm{~s}$, annealing at $59{ }^{\circ} \mathrm{C}$ for $30 \mathrm{~s}$ and extension at $68^{\circ} \mathrm{C}$ for $25 \mathrm{~s}$. The $430 \mathrm{bp}$ PCR products were directly sequenced using the ABI 3730xl DNA Analyzer instrument (Applied Biosystems) after purification from 1.5\% agarose gel. The 16-bp duplication polymorphism was determined by PCR using the forward primer: $5^{\prime}$-CGT TCTGGTAAGGACAAGGGTTGG-3' and reverse primer: 5'-AAAGAGCAGTCAGAGGACCAGGTC-3'. The reaction conditions were pre-denaturation at $94{ }^{\circ} \mathrm{C}$ for $2 \mathrm{~min}$; 30 cycles of denaturation at $98{ }^{\circ} \mathrm{C}$ for $10 \mathrm{~s}$, annealing at $59{ }^{\circ} \mathrm{C}$ for $30 \mathrm{~s}$ and extension at $68{ }^{\circ} \mathrm{C}$ for $6 \mathrm{~s}$. Then, PCR products were separated by $4 \%$ agarose gel 
and visualized by Gelred staining. Homozygous wild-type alleles (no duplication, designated Del allele) resulted in a $102 \mathrm{bp}$ fragment, while homozygous variant alleles (16 bp duplication, designated Ins allele) resulted in a $118 \mathrm{bp}$ fragment. Both two fragments $(102 \mathrm{bp}+118 \mathrm{bp})$ were obtained when the genotype was heterozygous Del/Ins. All reactions were performed in a total volume of $25 \mu \mathrm{L}$ mixture containing $20 \mathrm{ng}$ of gDNA and $0.3 \mu \mathrm{M}$ of each forward and reverse primer.

\section{Statistical analysis}

Statistical analysis was performed using SPSS version 19.0 (SPSS Inc., Chicago, USA) and the online HardyWeinberg equilibrium (HWE) calculator (http://ihg.gsf. de/cgi-bin/hw/hwa1.pl). Chi-square $\left(\chi^{2}\right)$ test was used to compare the categorical variables. Statistical significance level was set at 0.05 . The odds ratio (OR) with its 95\% confidence interval (CI) was calculated through logistic regression analysis (adjusted for age) to measure the association between genotypes of the two polymorphisms and breast cancer risk. Additionally, Armitage's trend test was also performed to improve the statistical power of this study.

\section{Results}

In this study, the distributions of the genotype frequencies of both codon 72 and intron 3 16-bp duplication polymorphisms among control group $(p=0.942$ and $p=0.407)$ were within Hardy-Weinberg equilibrium. For the codon 72 polymorphism, the heterozygous Arg/Pro was the most frequent genotype in both groups, and had a higher prevalence in breast cancer cases $\left(P_{\text {adj }}=0.10\right.$; $\mathrm{OR}_{\mathrm{adj}}=1.43$, 95\% CI 0.93-2.18). The distributions of Arg and Pro allele frequencies were almost equal in both groups $\left(P_{\mathrm{adj}}=0.52 ; \mathrm{OR}_{\mathrm{adj}}=1.09,95 \% \mathrm{CI} 0.84-1.42\right)$. Regarding the 16-bp duplication polymorphism, the homozygous Del/Del was the most frequent genotype in both groups and its distribution frequency was significantly higher (approximately eightfold) than that of the heterozygous Del/Ins; no homozygous 16-bp duplication (Ins/Ins) carrier was found in the whole 506 samples. Briefly, no statistically significant difference was obtained when analyzing using multiple genetic models (additive, dominant and recessive models) $\left(P_{\mathrm{adj}}>0.05\right)$ and Armitage's trend test $\left(P_{\text {trend }}=0.58\right.$ and 0.86$)$ (Table 2$)$. In addition, concerning the distributions of the genotype frequencies of the two polymorphisms in breast cancer patients with different clinicopathological features, no statistically significant difference was found as well $(p>0.05)$ (Additional file 1: Table S1).

Distributions of haplotypes of the two polymorphisms were further analyzed. For all haplotypes, no matter the most frequent Arg-Del or the rarest Arg-Ins, there was also no statistical significance $\left(P_{\text {adj }}>0.05\right)$ (Table 3$)$.

\section{Discussion}

Here, we investigated the relationship between two controversial TP53 polymorphisms codon 72 and intron 3 16-bp duplication as well as their haplotypes, and breast cancer risk in Chinese Han women. For the codon 72 polymorphism, a lot of studies have reported its contribution to the breast cancer susceptibility in women from different geographic areas and ethnic groups [8, 10, 11, 13]. Particularly, Siddique et al. found a strong correlation between the codon 72 Arg allele and susceptibility to Chinese breast cancer development [13]. In their study, the distribution of genotype frequencies of Arg/ Arg, Arg/Pro and Pro/Pro among breast cancer cases was $43.5 \%, 44.0 \%$ and $12.5 \%$, respectively; whereas in control group, this distribution was $35.0 \%, 45.0 \%$ and $20.0 \%$, respectively. In contrast to the high prevalence of homozygous Arg/Arg in the breast cancer cases and low prevalence of homozygous Pro/Pro in the controls, the frequency distribution of heterozygous Arg/Pro was almost equal between the two groups. On the contrary, many studies did not support this kind of positive correlation [16, 19, 22, 23]. For instance, Lum et al. found that the codon 72 polymorphism did not affect general breast cancer risk in Chinese women, but the Arg/Arg homozygote seemed to decrease the cancer risk in the later onset sporadic cases $(\mathrm{OR}=0.27,95 \% \mathrm{CI} 0.08-0.93$; adjusted for age), which was inconsistent with Siddique's findings [16]. However, in this study, the results were a little different from those reported by both Siddique and Lum. The distribution frequencies of both Arg/Arg and Pro/Pro homozygotes in breast cancer cases were lower than those among healthy controls; by contrast, the Arg/ Pro heterozygote accounted for a larger proportion in both groups, and was more frequent in breast cancer cases, which implied that it might exist as the possible risk genotype of the codon 72 polymorphism despite that there was not any statistical significant correlation under the three genetic models (Table 2). The association has been proved to be more evident in Caucasians living in Europe and America than Chinese Han women. The heterozygous RP but not homozygous RR which relates to increased incidence of breast cancer indicates that this variation had an extremely tiny influence on the function of TP53. Furthermore, no effect of any genotype of the codon 72 polymorphism on breast cancer cases with different clinicopathological features could be observed in this study as well, no matter the later-onset cases or early-onset cases $(p=0.85)$ (Additional file 1: Table S1). These contradictory results were probably caused by the bias of sample collection, such as smaller sample size of 
Table 2 Distribution of genotype and allele frequencies of TP53 codon 72 and 16-bp duplication polymorphisms in breast cancer cases and controls

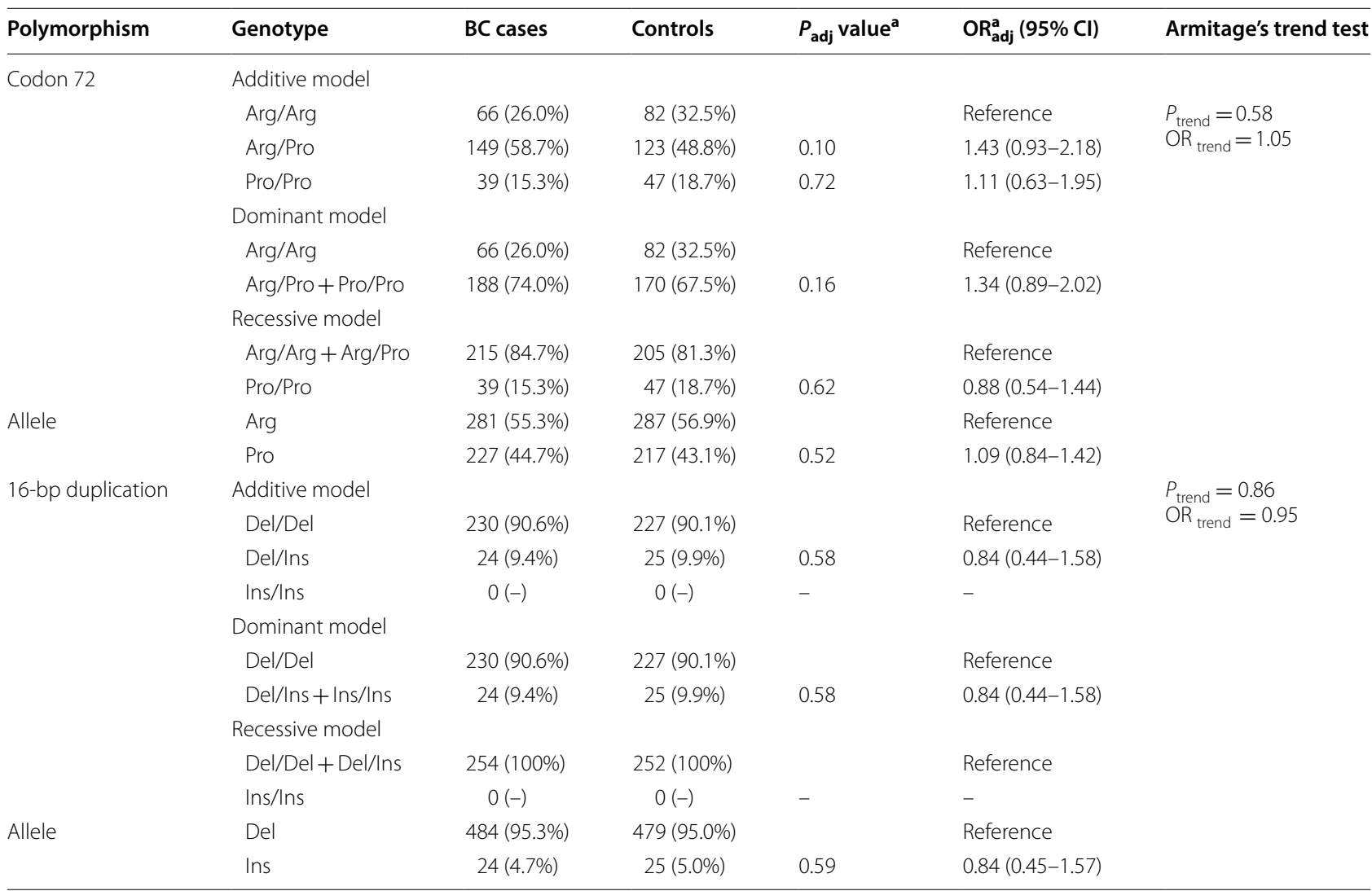

$B C$ cases breast cancer cases, $O R$ odds ratio, $\mathrm{Cl}$ confidence interval

adjusted by age

Table 3 Analysis of haplotype frequencies of TP53 codon 72 and 16-bp duplication polymorphisms in breast cancer cases and controls

\begin{tabular}{lllll}
\hline Haplotype & $\mathbf{B C}$ cases & Controls & $\boldsymbol{P}_{\text {adj }}$-value & $\mathbf{O R}_{\text {adj }}(\mathbf{9 5} \% \mathbf{C l})$ \\
\hline Arg-Del & 0.48 & 0.49 & & Reference \\
Pro-Del & 0.42 & 0.41 & 0.67 & $1.07(0.79-1.44)$ \\
Arg-Ins & 0.05 & 0.04 & 0.90 & $1.05(0.51-2.17)$ \\
Pro-Ins & 0.05 & 0.06 & 0.65 & $0.86(0.45-1.64)$ \\
\hline
\end{tabular}

either breast cancer case group (Siddique's study, $N=94$ ) or control group (Lum's study, $N=80$ ) of their studies.

Regarding the 16-bp duplication polymorphism, most of the previous studies accepted that the Ins allele, especially homozygous Ins/Ins, contributed to the increase of breast cancer risk [8, 11, 24-26]. However, we could not find any Ins/Ins homozygote carrier in the total 506 samples, implying that this risk genotype may not affect breast cancer risk in Chinese women because of its extremely low prevalence. In terms of the distribution of heterozygous Del/Ins as well as Ins allele frequencies, no statistical significant difference was found between the two groups (Table 2). These indicate that the intron 316 -bp duplication is not likely to correlate with the development of breast cancer.

On the other hand, the haplotypes of the two polymorphisms may be another important factor affecting breast cancer risk, since it was reported that they were in strong linkage disequilibrium [38]. Some studies have also gained positive results to support this hypothesis, such as Costa et al. found that the Arg-Ins had correlation with breast cancer risk [11], whereas Osorio et al. found a converse result that the Pro-Del was associated with an earlier age at the onset of the first primary tumor [23]. However, just as the codon 72 polymorphism, which kind of haplotype is indeed the risk factor of the breast cancer progression remains to be seen. In this study, no significant association was found between the haplotypes and Chinese breast 
cancer risk, no matter the mentioned Arg-Ins, Pro-Del, or the other two kinds of haplotypes (Table 3).

\section{Conclusion}

In conclusion, through a population-based case-control study, we found that there was no significant association between TP53 codon 72 as well as intron 3 16-bp duplication polymorphisms and breast cancer risk in Chinese Han women when multiple genetic models along with Armitage's trend test were utilized in the analysis, but the heterozygous Arg/Pro may exist as the possible risk genotype of the codon 72 polymorphism, which were a little distinguished from the conclusions of previous studies. Still, more relevant studies designed for Chinese population, especially those with larger sample size, need to be done to further validate these findings.

\section{Additional file}

Additional file 1: Table S1. Distributions of the genotype frequencies of codon 72 and intron 3 16-bp duplication polymorphisms in breast cancer patients with different clinicopathological features.

\section{Abbreviations}

SNPs: single nucleotide polymorphisms; OR: odds ratio; Cl: confidence interval.

\section{Authors' contributions}

XX participated in the design of present study and drafted the manuscript. $X C$ and $C Z$ designed the research and wrote the manuscript. XC collected the materials of the patients. WH performed the laboratory test. All authors read and approved the final manuscript.

\section{Author details}

${ }^{1}$ Institute of Life Sciences, Jiangsu University, Zhenjiang, China. ${ }^{2}$ Pathogen Diagnostic Center, Institut Pasteur of Shanghai, Chinese Academy of Sciences, Shanghai, China. ${ }^{3}$ Department of Chemotherapy, Jiangsu Cancer Hospital, Jiangsu Institute of Cancer Research, Nanjing Medical University Affiliated Cancer Hospital, Nanjing 210009, Jiangsu, People's Republic of China. ${ }^{4}$ Department of Gynecologic Oncology, Jiangsu Cancer Hospital, Jiangsu Institute of Cancer Research, Nanjing Medical University Affiliated Cancer Hospital, 42\# Baiziting Street, Nanjing 210009, Jiangsu, People's Republic of China.

\section{Acknowledgements}

We thank Ci Chu (Institute of Life Sciences, Jiangsu University, China) for his kind help in data processing and analysis.

\section{Competing interests}

The authors declare that they have no competing interests.

\section{Availability of data and materials}

We would not share the data and material used in this manuscript, because we need them for further research.

\section{Consent for publication}

Not applicable.

\section{Ethics approval and consent to participate}

This retrospective study was approved by the institutional review board of Jiangsu Cancer Hospital, Nanjing Medical University, China. The informed consent requirement was waived. The committee's reference number was Ethical Committee of Nanjing Medical University 2017-405.

\section{Funding}

This study was supported by grants from the National Natural Science Foundation of China (Nos. 81472441, 31271272); six major talent summit (No. 2013wsn-62); Natural Science Foundation of Jiangsu Province (No. BK20131439).

\section{Publisher's Note}

Springer Nature remains neutral with regard to jurisdictional claims in published maps and institutional affiliations.

Received: 4 July 2016 Accepted: 25 September 2018

Published online: 11 October 2018

\section{References}

1. Anderson BO. Breast cancer-thinking globally. Science. 2014;343(6178):1403.

2. Walerych $\mathrm{D}$, et al. The rebel angel: mutant p53 as the driving oncogene in breast cancer. Carcinogenesis. 2012;33(11):2007-17.

3. Vogelstein B, Lane D, Levine AJ. Surfing the p53 network. Nature. 2000;408(6810):307-10.

4. Vousden KH, Lane DP. p53 in health and disease. Nat Rev Mol Cell Biol. 2007;8(4):275-83.

5. Whibley C, Pharoah PD, Hollstein M. p53 polymorphisms: cancer implications. Nat Rev Cancer. 2009;9(2):95-107.

6. Kaur S, et al. Analysis of TP53 polymorphisms in North Indian sporadic esophageal cancer patients. Asian Pac J Cancer Prev. 2014;15(19):8413-22.

7. Malisic E, et al. TP53 codon 72 polymorphism and risk of cervical carcinoma in Serbian women. Arch Gynecol Obstet. 2013;288(3):621-5.

8. Sharma S, et al. TP53 polymorphisms in sporadic North Indian breast cancer patients. Asian Pac J Cancer Prev. 2014;15(16):6871-9.

9. Kafshdooz $\mathrm{T}$, et al. Polymorphism of p53 gene codon 72 in endometrial cancer: correlation with tumor grade and histological type. Asian Pac J Cancer Prev. 2014;15(22):9603-6.

10. Damin AP, et al. Evidence for an association of TP53 codon 72 polymorphism with breast cancer risk. Cancer Detect Prev. 2006;30(6):523-9.

11. Costa S, et al. Importance of TP53 codon 72 and intron 3 duplication 16 bp polymorphisms in prediction of susceptibility on breast cancer. BMC Cancer. 2008;8:32.

12. Zhang Z, et al. P53 codon 72 polymorphism contributes to breast cancer risk: a meta-analysis based on 39 case-control studies. Breast Cancer Res Treat. 2010;120(2):509-17.

13. Siddique $\mathrm{MM}$, et al. Evidence for selective expression of the $\mathrm{p} 53$ codon 72 polymorphs: implications in cancer development. Cancer Epidemiol Biomarkers Prev. 2005;14(9):2245-52.

14. Moradi MT, et al. Effects of p53 codon 72 and MDM2 SNP309 polymorphisms on gastric cancer risk among the Iranian population. Asian Pac J Cancer Prev. 2014;15(17):7413-7.

15. Saikia BJ, et al. Association of a p53 codon 72 gene polymorphism with environmental factors and risk of lung cancer: a case control study in Mizoram and Manipur, a high incidence region in North East India. Asian Pac J Cancer Prev. 2014;15(24):10653-8.

16. Lum SS, et al. MDM2 SNP309 G allele increases risk but the T allele is associated with earlier onset age of sporadic breast cancers in the Chinese population. Carcinogenesis. 2008;29(4):754-61.

17. Liu T, et al. Genetic association between p53 codon 72 polymorphism and risk of cutaneous squamous cell carcinoma. Tumour Biol. 2014;35(4):3899-903.

18. Ma Y, et al. No significant association between the TP53 codon 72 polymorphism and breast cancer risk: a meta-analysis of 21 studies involving 24,063 subjects. Breast Cancer Res Treat. 2011;125(1):201-5.

19. Gohari-Lasaki S, et al. Lack of influence of TP53 Arg72Pro and 16 bp duplication polymorphisms on risk of breast cancer in Iran. Asian Pac J Cancer Prev. 2015;16(7):2971-4

20. Laprano TD, et al. Association of TP53 codon 72 and intron 3 16-bp Ins/Del polymorphisms with cervical cancer risk. Tumour Biol. 2014;35(8):7435-40 
21. Chang Z, Yu X. Association between p53 codon 72 polymorphism and sarcoma risk among Caucasians. Tumour Biol. 2014;35(5):4807-12.

22. Cavallone L, et al. Haplotype analysis of TP53 polymorphisms, Arg72Pro and Ins16, in BRCA1 and BRCA2 mutation carriers of French Canadian descent. BMC Cancer. 2008;8:96.

23. Osorio A, et al. An evaluation of the polymorphisms Ins 16 bp and Arg72Pro in p53 as breast cancer risk modifiers in BRCA1 and BRCA2 mutation carriers. Br J Cancer. 2008;99(6):974-7.

24. Gemignani F, et al. A TP53 polymorphism is associated with increased risk of colorectal cancer and with reduced levels of TP53 mRNA. Oncogene. 2004;23(10):1954-6.

25. Wu D, et al. Intron 3 sixteen base pairs duplication polymorphism of p53 contributes to breast cancer susceptibility: evidence from meta-analysis. PLOS ONE. 2013;8(4):e61662.

26. Hu Z, et al. Intron 316 bp duplication polymorphism of TP53 contributes to cancer susceptibility: a meta-analysis. Carcinogenesis. 2010;31(4):643-7.

27. Zheng W, et al. Common genetic determinants of breast-cancer risk in East Asian women: a collaborative study of 23637 breast cancer cases and 25579 controls. Hum Mol Genet. 2013:22(12):2539-50.

28. Wen $W$, et al. Prediction of breast cancer risk based on common genetic variants in women of East Asian ancestry. Breast Cancer Res. 2016;18(1):124.

29. Wang $Y$, et al. Evaluation of functional genetic variants at $6 q 25.1$ and risk of breast cancer in a Chinese population. Breast Cancer Res. 2014;16(4):422.
30. Li H, et al. Gene-environment interactions for breast cancer risk among Chinese women: a report from the Shanghai Breast Cancer Genetics Study. Am J Epidemiol. 2012;177(2):161-70.

31. Barzan D, et al. Comparison of genetic variation of breast cancer susceptibility genes in Chinese and German populations. Eur J Hum Genet. 2013;21(11):1286-92.

32. Dai J, et al. Breast cancer risk assessment with five independent genetic variants and two risk factors in Chinese women. Breast Cancer Res. 2012;14(1):R17.

33. Zheng W, et al. Genetic and clinical predictors for breast cancer risk assessment and stratification among Chinese women. J Natl Cancer Inst. 2010;102(13):972-81.

34. Low S-K, et al. Genome-wide association study of breast cancer in the Japanese population. PLoS ONE. 2013:8(10):e76463.

35. Han W, et al. Common genetic variants associated with breast cancer in Korean women and differential susceptibility according to intrinsic subtype. Cancer Epidemiol Prev Biomarkers. 2011;20(5):793-8.

36. Bhoo-Pathy $\mathrm{N}$, et al. Breast cancer research in Asia: adopt or adapt Western knowledge? Eur J Cancer. 2013;49(3):703-9.

37. Long J, et al. Genome-wide association study in east Asians identifies novel susceptibility loci for breast cancer. PLoS Genet. 2012;8(2):e1002532

38. Birgander R, et al. P53 polymorphisms and haplotypes in lung cancer. Carcinogenesis. 1995;16(9):2233-6.
Ready to submit your research? Choose BMC and benefit from:

- fast, convenient online submission

- thorough peer review by experienced researchers in your field

- rapid publication on acceptance

- support for research data, including large and complex data types

- gold Open Access which fosters wider collaboration and increased citations

- maximum visibility for your research: over $100 \mathrm{M}$ website views per year

At $\mathrm{BMC}$, research is always in progress.

Learn more biomedcentral.com/submissions 